\title{
DØ EVIDENCE FOR CP VIOLATION AND IMPLICATION FOR CPT VIOLATION IN B-MESON MIXING
}

\author{
R. VAN KOOTEN* FOR THE D $\varnothing$ COLLABORATION** \\ Department of Physics, Indiana University \\ Bloomington, Indiana 47405, USA \\ *E-mail: rvankoot@indiana.edu \\ ${ }^{* *}$ http://www-d0.fnal.gov
}

\begin{abstract}
A D $\varnothing$ analysis measuring the charge asymmetry $A_{\mathrm{sl}}^{b}$ of like-sign dimuon events due to semileptonic $b$-hadron decays at the Fermilab Tevatron Collider is described. It differs by 3.2 standard deviations from the Standard Model prediction to provide first evidence of CPT-invariant anomalous $\mathrm{CP}$ violation in the mixing of neutral $B$ mesons, and is compared to the CP-violating phase obtained from a $\mathrm{D} \varnothing$ analysis of the time-dependent decay angles in $B_{s}^{0} \rightarrow J / \psi \phi$. If CPT violation is allowed, the dimuon asymmetry also yields the first sensitivity to CPT violation in the $B_{s}^{0}$ system.
\end{abstract}

\section{Introduction}

The interferometric systems of the particle-antiparticle oscillations of neutral mesons are particularly sensitive to testing for $\mathrm{CP}$ and $\mathrm{CPT}$ violation. $\mathrm{CP}$ violation has been observed at small levels in a number of these systems, ${ }^{1}$ and the violation of CP symmetry is a necessary condition for the matter-antimatter asymmetry of our universe ${ }^{2}$ and for our very existence. However, the observed levels of CP violation in the $K^{0}$ and $B_{d}^{0}$ systems are not large enough to account for this asymmetry, implying the need for additional sources of CP violation beyond the Standard Model (SM). Both CP and $\mathrm{CPT}$ violation in the neutral $B$-meson system are considered below.

\section{2. $\mathrm{D} \emptyset$ dimuon charge asymmetry}

In $6.1 \mathrm{fb}^{-1}$ of $p \bar{p}$ collision data, the DØ Collaboration first measures ${ }^{3}$ the raw dimuon charge asymmetry $A=\left(N^{\mu^{+} \mu^{+}}-N^{\mu^{-} \mu^{-}}\right) /\left(N^{\mu^{+} \mu^{+}}+N^{\mu^{-} \mu^{-}}\right)$ regardless of muon source. From pure physics processes at the primary interaction, one of the very few sources of same-sign dileptons in the same collision event is due to $B$ physics. If there is a nonzero asymmetry after 
correcting for backgrounds, the assumption is that it is coming from neutral $B$-meson mixing, i.e., the dimuon charge asymmetry of semileptonic $B$ decays $A_{\mathrm{sl}}^{b}=\left(N_{b}^{\mu^{+} \mu^{+}}-N_{b}^{\mu^{-} \mu^{-}}\right) /\left(N_{b}^{\mu^{+} \mu^{+}}+N_{b}^{\mu^{-} \mu^{-}}\right)$. The great majority of $b$ quark production at the Tevatron is via $b \bar{b}$, so this can occur, for example, when the $b$ quark decays semileptonically directly $\bar{B}_{q}^{0} \rightarrow \mu^{-}$, but for the $\bar{b}$ quark, there is first a $B$-meson oscillation before the semileptonic decay, i.e., $B_{q}^{0} \rightarrow \bar{B}_{q}^{0} \rightarrow \mu^{-}$. Another way to measure this asymmetry is via inclusive 'wrong-sign' decays, i.e., $\bar{B} \rightarrow \mu^{+} X$ which is only possible through flavor oscillation of $B_{d}^{0}$ and $B_{s}^{0}$. A semileptonic charge asymmetry can then be constructed:

$$
a_{\mathrm{sl}}^{b}=\frac{\Gamma\left(\bar{B} \rightarrow \mu^{+}\right)-\Gamma\left(B \rightarrow \mu^{-} X\right)}{\Gamma\left(\bar{B} \rightarrow \mu^{+}\right)+\Gamma\left(B \rightarrow \mu^{-} X\right)}
$$

and probed by measuring the inclusive raw single muon asymmetry $a=$ $\left(n^{\mu^{+}}-n^{\mu^{-}}\right) /\left(n^{\mu^{+}}+n^{\mu^{-}}\right)$. Assuming CPT symmetry holds, it can be shown ${ }^{4}$ that $A_{\mathrm{sl}}^{b}=a_{\mathrm{sl}}^{b}$. Both the raw asymmetries contain contributions from $A_{\mathrm{sl}}^{b}$, other processes producing muons, plus detector related backgrounds. The background contributions to the asymmetries are mostly determined by independent measurements in the data, with minimal input from simulation. Monte Carlo simulation is only used to determine the remaining fraction of like-sign dimuons, and fraction of single muons from mixed $b$-hadron decays. The raw asymmetry $a$ is dominated by backgrounds, and the different signal and correlated background content of both asymmetries is used to minimize the total uncertainty on $A_{\mathrm{sl}}^{b}$.

From $1.5 \times 10^{9}$ muons in the inclusive sample, $a=(0.955 \pm 0.003) \%$, and from $3.7 \times 10^{6}$ events in the like-sign dimuon sample, $A=(0.564 \pm 0.053) \%$. The most important detector-related background is having one muon from a semileptonic $b$-hadron decay, and the other from a decay in flight of $K \rightarrow \mu \nu$ and $\pi \rightarrow \mu \nu$, subsequent punch-through to the muon detectors (from showers in the material of the calorimeters), and sail-through of $\pi, K$ and $p$, where these hadrons are either from the other $b$-hadron decay or from fragmentation. The polarities of the $\mathrm{D} \varnothing$ detector toroid and solenoid magnets are switched every two weeks during data running, so that residual muon reconstruction charge asymmetries cancel to first order. This reduces these background asymmetries from $\mathcal{O}(3 \%)$ to less than $0.1 \%$. Without this capability, the systematic uncertainty on the final asymmetry would be substantially larger.

From the like-sign dimuon sample, after correcting for backgrounds,

$$
A_{\mathrm{sl}}^{b}=-0.00736 \pm 0.00266 \text { (stat) } \pm 0.00305 \text { (syst). }
$$


The corrected asymmetry from the inclusive single muon sample is used to constrain the backgrounds in the dimuon sample and a linear combination chosen to minimize the total uncertainty on $A_{\mathrm{sl}}^{b}$ arriving at:

$$
A_{\mathrm{sl}}^{b}=-0.00957 \pm 0.00251 \text { (stat) } \pm 0.00146 \text { (syst). }
$$

This result is 3.2 standard deviations away from the SM prediction for CPT-preserving $\mathrm{T}$ violation, which is ${ }^{5} A_{\mathrm{sl}}^{b}(\mathrm{SM})=\left(-2.3_{-0.6}^{+0.5}\right) \times 10^{-4}$ and represents the first evidence for anomalous $\mathrm{CP}$ violation in the mixing of neutral $B$ mesons.

\section{Comparison to CP-violating phase in $B_{s}^{0}$ system}

The asymmetry above has contributions from both $B_{d}^{0}$ and $B_{s}^{0}$ oscillations, and using world-average values of mixing parameters, ${ }^{1,3}$

$$
A_{\mathrm{sl}}^{b}=(0.506 \pm 0.043) a_{\mathrm{sl}}^{d}+(0.494 \mp 0.043) a_{\mathrm{sl}}^{s} .
$$

If the world-average ${ }^{1}$ value of $a_{\mathrm{sl}}^{d}$ from the $B$ factories running at the $\Upsilon(4 S)$ is input to the above, the value $a_{\mathrm{sl}}^{s}=(-1.46 \pm 0.75) \%$ is obtained, less than three standard deviations from the SM prediction ${ }^{5}$ of $a_{\mathrm{sl}}^{s}(S M)=(-0.0021 \pm$ $0.0006) \%$ due to the uncertainty on the coefficients of Eq. (4). However, this still provides an interesting comparison to a $\mathrm{D} \varnothing$ result $^{6}$ where $\mathrm{CP}$ violation is probed by the time-dependent angular analysis of decay products in $B_{s}^{0} \rightarrow J / \psi \phi$ where there is interference between the diagrams with and without $\left(\bar{B}_{s}^{0} \rightarrow J / \psi \phi\right)$ mixing. The connection is through the expression ${ }^{5}$ $a_{\mathrm{sl}}^{s}=\left(\Delta \Gamma_{s} / \Delta M_{s}\right) \tan \phi_{s}$ where $\Delta \Gamma_{s}$ and $\Delta M_{s}$ are the width and mass differences between mass eigenstates for the $B_{s}^{0}$, and $\phi_{s}$ is the measured $\mathrm{CP}$-violating phase angle between the complex off-diagonal mixing-matrix elements $\Gamma_{s}^{12}$ and $M_{s}^{12}$. The comparison shown in Fig. 1 shows an intriguing similar trend in difference from the SM prediction of the two analyses.

\section{Implications for CPT violation}

A CPT-violating effect in $B$-meson mixing was predicted some time ago ${ }^{7}$ as potentially arising from spontaneous breaking of Lorentz symmetry in an underlying unified theory, ${ }^{8}$ and the $B_{s}^{0}-\bar{B}_{s}^{0}$ system is of particular interest for studies of $\mathrm{CPT}$ violation because several complete particle-antiparticle oscillations occur within a meson lifetime. ${ }^{9}$ Working within the comprehensive effective field theory describing general Lorentz violation at attainable energies known as the Standard-Model Extension (SME), ${ }^{10}$ each 

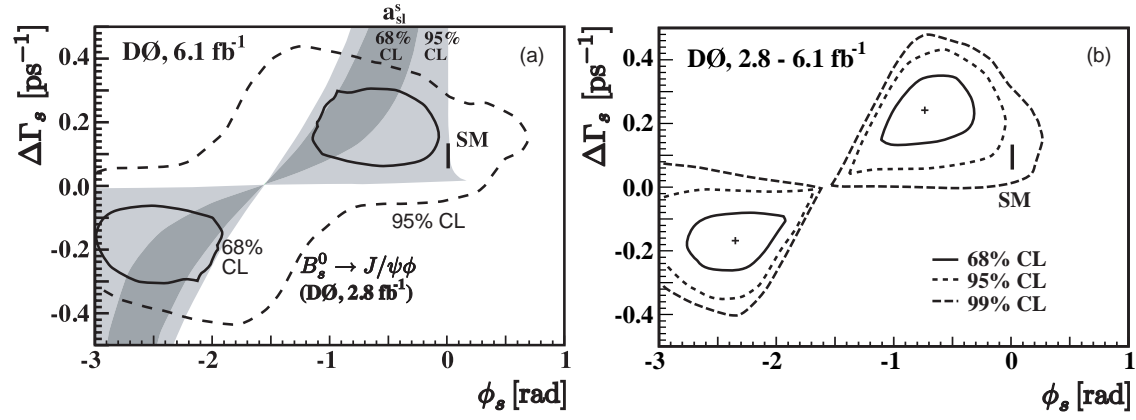

Fig. 1. (a) The $68 \%$ and $95 \%$ C.L. regions (shaded bands) of probability for $\Delta \Gamma_{s}$ and $\phi_{s}$ from the dimuon asymmetry compared to the regions (solid and dashed lines) from the $B_{s}^{0} \rightarrow J / \psi \phi$ measurement. (b) Combination of the two results and resulting C.L. regions. The SM prediction for these parameters is also shown for both cases.

CPT-violating term in the SME Lagrange density is the product of a CPTviolating operator and a controlling coefficient, and in this case a combination of the four SME coefficients ${ }^{11}\left(\Delta a^{B_{s}}\right)_{\mu}$.

For these purposes, the asymmetry using single inclusive muons of Ref. 3 and the combined asymmetry of Eq. (4) are irrelevant, and only the first asymmetry $A_{\mathrm{sl}}^{b}$ result of Eq. (2) is considered. A measure of CPT violation is given by the inclusive 'right-charge' muon charge asymmetry $\mathcal{A}_{\mathrm{CPT}}^{b}$ of semileptonic decays of $b$ hadrons,

$$
\mathcal{A}_{\mathrm{CPT}}^{b}=\frac{\Gamma\left(\bar{B} \rightarrow \mu^{-} X\right)-\Gamma\left(B \rightarrow \mu^{+} X\right)}{\Gamma\left(\bar{B} \rightarrow \mu^{-} X\right)+\Gamma\left(B \rightarrow \mu^{+} X\right)} .
$$

In terms of this CPT asymmetry and the T asymmetry of Eq. (1),

$$
A_{\mathrm{sl}}^{b}=\left(\frac{1+a_{\mathrm{sl}}^{b}}{1-a_{\mathrm{sl}}^{b}}-\frac{1+\mathcal{A}_{\mathrm{CPT}}^{b}}{1-\mathcal{A}_{\mathrm{CPT}}^{b}}\right) /\left(\frac{1+a_{\mathrm{sl}}^{b}}{1-a_{\mathrm{sl}}^{b}}+\frac{1+\mathcal{A}_{\mathrm{CPT}}^{b}}{1-\mathcal{A}_{\mathrm{CPT}}^{b}}\right) \approx a_{\mathrm{sl}}^{b}-\mathcal{A}_{\mathrm{CPT}}^{b} \cdot(6)
$$

Assuming the only source of $\mathrm{T}$ violation is the SM contribution $a_{\mathrm{sl}}^{b}(\mathrm{SM})=$ $A_{\mathrm{sl}}^{b}(\mathrm{SM})$, combining with the $\mathrm{D} \varnothing$ dimuon asymmetry of Eq. (2) results in

$$
\mathcal{A}_{\mathrm{CPT}}^{b}=0.00713 \pm 0.00405 \text {. }
$$

To interpret this as a measure of CPT violation in $B$-meson mixing, and in particular in the $B_{s}^{0}-\bar{B}_{s}^{0}$ system, the $\omega \xi$ formalism ${ }^{11}$ is adopted that allows for CPT violation of arbitrary size, governed by a complex parameter $\xi$ of arbitrary size. The complex parameter $\xi$ cannot be a scalar since CPT violation comes with Lorentz violation. ${ }^{12}$ It must depend on the $B$-meson four-momentum and is therefore a frame-dependent quantity. The rotation of the Earth relative to the constant vector $\Delta \vec{a}$ generates a variation with 
sidereal time in $\xi$, but it is possible to average over the sidereal time and the meson four-momentum. Since the particle distributions from $b$-hadron decay for the Tevatron collider are symmetric in local detector polar coordinates for $\mathrm{D} \emptyset$, the dependence on the spatial components $\left(\Delta a^{B_{s}}\right)_{J}$ cancels. Details of the relationship between the asymmetry $\mathcal{A}_{\mathrm{CPT}}^{b}$, the parameter $\xi_{s}$ specific for the $B_{s}^{0}-\bar{B}_{s}^{0}$ system, and the time component SME coefficient $\left(\Delta a^{B_{s}}\right)_{T}$ can be found in Ref. 13. Assuming that the only source of CPT violation comes from $B_{s}^{0}-\bar{B}_{s}^{0}$ mixing, the value

$$
\left(\Delta a^{B_{s}}\right)_{T}=(3.7 \pm 3.8) \times 10^{-12} \mathrm{GeV},
$$

is found. This corresponds to the bound

$$
-3.8 \times 10^{-12}<\left(\Delta a^{B_{s}}\right)_{T}<1.1 \times 10^{-11}
$$

at the $95 \%$ confidence level. The value of Eq. (8), documented in Ref. 13, represents the first sensitivity to CPT violation in the $B_{s}^{0}-\bar{B}_{s}^{0}$ system.

\section{Acknowledgments}

The DØ Collaboration thanks the staffs at Fermilab and collaborating institutions, and acknowledge support from agencies including the DOE and NSF (USA).

\section{References}

1. C. Amsler et al., Phys. Lett. B 667, 1 (2008), and 2009 partial update for the 2010 edition, and references therein.

2. A.D. Sakharov, Sov. Phys. JETP Lett. 5, 24 (1967); P. Huet and E. Sather, Phys. Rev. D 51, 379 (1995).

3. V.M. Abazov et al. (DØ Collaboration), Phys. Rev. D, in press [arXiv:1005.2757]; V.M. Abazov et al. (DØ Collaboration), Phys. Rev. Lett., in press [arXiv:1007.0395].

4. Y. Grossman, Y. Nir, and G. Raz, Phys. Rev. Lett. 97, 151801 (2006).

5. A. Lenz and U. Nierste, J. High Energy Phys. 0706, 072 (2007).

6. V.M. Abazov et al. (DØ Collaboration), Phys. Rev. Lett. 101, 241801 (2008).

7. V.A. Kostelecký and R. Potting, Phys. Rev. D 51, 3923 (1995).

8. V.A. Kostelecký and S. Samuel, Phys. Rev. D 39, 683 (1989); V.A. Kostelecký and R. Potting, Nucl. Phys. B 359, 545 (1991).

9. DØ Collaboration, V.M. Abazov et al., Phys. Rev. Lett. 97, 021802 (2006); CDF Collaboration, A. Abulencia et al., Phys. Rev. Lett. 97, 242003 (2006).

10. D. Colladay and V.A. Kostelecký, Phys. Rev. D 55, 6760 (1997); Phys. Rev. D 58116002 (1998); V.A. Kostelecký, Phys. Rev. D 69, 105009 (2004).

11. V.A. Kostelecký, Phys. Rev. D 64, 076001 (2001).

12. O.W. Greenberg, Phys. Rev. Lett. 89, 231602 (2002).

13. V.A. Kostelecký and R. Van Kooten, arXiv:1007.5312. 